\title{
Development of a novel DDS for site-specific PEGylated proteins
}

Yasuo Yoshioka ${ }^{1,2,3}$, Shin-ichi Tsunoda ${ }^{2,3,4^{*}}$ and Yasuo Tsutsumi ${ }^{1,2,3^{*}}$

\begin{abstract}
Because of the shifted focus in life science research from genome analyses to genetic and protein function analyses, we now know functions of numerous proteins. These analyses, including those of newly identified proteins, are expected to contribute to the identification of proteins of therapeutic value in various diseases. Consequently, pharmacoproteomic-based drug discovery and development of protein therapies attracted a great deal of attention in recent years. Clinical applications of most of these proteins are, however, limited because of their unexpectedly low therapeutic effects, resulting from the proteolytic degradation in vivo followed by rapid removal from the circulatory system. Therefore, frequent administration of excessively high dose of a protein is required to observe its therapeutic effect in vivo. This often results in impaired homeostasis in vivo and leads to severe adverse effects. To overcome these problems, we have devised a method for chemical modification of proteins with polyethylene glycol (PEGylation) and other water-soluble polymers. In addition, we have established a method for creating functional mutant proteins (muteins) with desired properties, and developed a site-specific polymer-conjugation method to further improve their therapeutic potency. In this review, we are introducing our original protein-drug innovation system mentioned above.
\end{abstract}

\section{Introduction}

The success of the human genome project has clearly shown that the human genome encodes about 35,000 genes and over half of them are poorly understood proteins. A number of these proteins are thought to be related to pathological disorders and other biological phenomena, and thus, are potentially useful as therapeutic agents and as targets for pharmaceutical development. Therefore, focus in life science research has currently shifted to newer fields, such as proteomics and structural genomics, in which the function and structure of proteins are analyzed en masse. Such analyses of large number of proteins, including the newly identified proteins, are expected to contribute to the identification of proteins of therapeutic importance in various diseases. In recent years, protein therapies using cytokines or antibodies have attracted a great deal of attention. Indeed, attempts are currently under progress to

\footnotetext{
* Correspondence: tsunoda@nibio.go.jp; ytsutsumi@phs.osaka-u.ac.jp 'Department of Toxicology and Safety Science, Graduate School of Pharmaceutical Sciences, Osaka University, 1-6 Yamadaoka, Suita, Osaka 5650871, Japan

${ }^{2}$ The Center for Advanced Medical Engineering and Informatics, Osaka University, 1-6, Yamadaoka, Suita, Osaka 565-0871, Japan Full list of author information is available at the end of the article
}

develop a wide range of therapeutic proteins for treating various diseases including cancer, hepatitis and autoimmune conditions[1-4]. Unfortunately, clinical applications of many of these proteins are limited because of their unexpectedly poor therapeutic effects[5,6]. Often these proteins are degraded by various proteases in vivo and rapidly removed from the circulatory system. Consequently, frequent administration of an excessively high dose of a protein is required to obtain its desired therapeutic effect in vivo, leading to a disturbance in the homeostasis and unexpected side effects. Additionally, bioactive proteins, such as cytokines, generally show pleiotropic actions through a number of receptors in vivo, making it difficult to elicit the desired effect without simultaneously triggering undesirable secondary effects. From this standpoint, creation of novel technologies that overcome the problems peculiar to bioactive proteins is essential for the advancement of pharmacoproteomic-based drug development. These technologies are suitable as Drug Delivery Systems (DDSs), aiming to maximize the therapeutic potency of proteins.

Our laboratory aims to develop novel DDS techniques for overcoming the problems of protein therapy: (i) establishment of a novel polymer-conjugation system to 
dramatically improve in vivo stability and selectively of bioactive proteins (polymeric DDS) and (ii) development of a powerful system to rapidly create functional mutant proteins (muteins) with enhanced receptor affinity and receptor specificity using a phage display technique (biological DDS). We are currently in the process of combining both approaches to create a protein-drug innovation system to further promote pharmaco-proteomic-based drug development. In this review, we will describe DDS-based technology to create functional mutants for advanced medical applications using the tumor necrosis factor-alpha (TNF) as an example and the usefulness of site-specific polymer-conjugation of proteins.

\section{Bioconjugation as a polymeric DDS}

One way to circumvent the problems of protein therapy is to conjugate them with polyethylene glycol (PEG) and other water-soluble polymeric modifiers (Figure 1) $[4,7,8]$. The covalent conjugation of proteins with PEG (PEGylation) increases their molecular size and steric hindrance, both of which depend on the properties of the PEG attached to the protein. Bioconjugation of proteins decreases their renal excretion rate due to the increased molecular size. In addition, because the polymers cover the protein surface, attack by a protease is generally blocked due to the steric hindrance, thus prolonging the half-life of the protein in vivo. The prolonged circulating lifetime in blood induces the enhanced permeability and retention (EPR) effect, which is based on the leaky nature of the tumor blood vessels, resulting in increased delivery of the conjugates to the tumor tissue[9]. Because of all these advantages, it is possible to use the bioactive protein at a decreased dose. However, this approach is limited by the frequent substantial loss of protein specific activity associated with polymer-conjugation. Bioconjugation commonly targets the $\varepsilon$-amino group of lysine residues and/or the $\mathrm{N}$-terminal $\alpha$-amino group of the protein because they are highly reactive and the bionconjugation reaction is mild

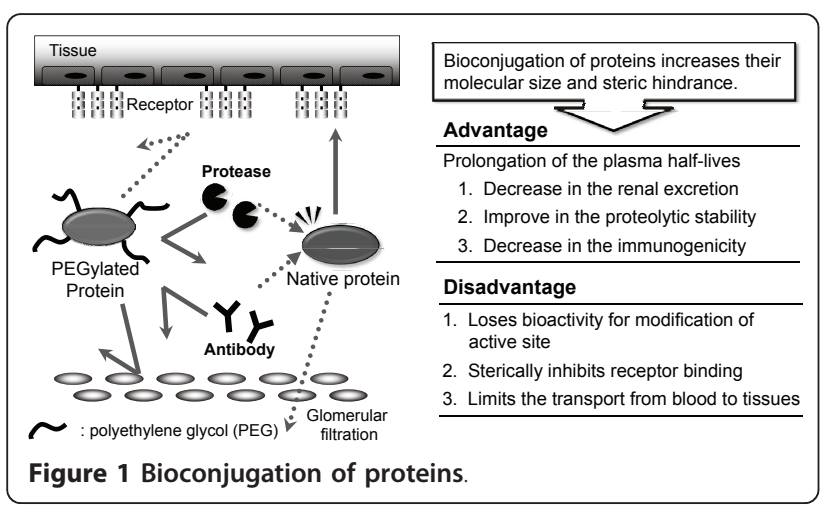

enough to minimize disruption of the protein structure. Because lysine residues often assume important roles in the formation of multi-dimensional structures and in bonding between ligands and receptors, introduction of polymers at these sites can potentially reduce the biological activity of the protein[10,11]. Indeed, PEGylated interferon, which has raised hopes as a potential cure for hepatitis $\mathrm{C}$, can only be produced as a heterogeneous mixture with $10-30 \%$ of the anticipated activity[11].

Tumor necrosis factor- $\alpha$ (TNF- $\alpha$ ), an antitumor cytokine, has numerous bioactivities including direct cytotoxicity against tumor cells, activation of immune antitumor response, and selective impairment of tumorblood vessels[12]. Thus, TNF- $\alpha$ has been considered as a novel antitumor agent[13-15]. However, as a systemic antitumor agent, very high doses of TNF- $\alpha$ were required to obtain sufficient clinical responses, because TNF- $\alpha$ is rapidly cleared from the circulation and also because it becomes widely distributed to various tissues following intravenous administration. As a result, TNF$\alpha$ with pleiotropic in vivo actions exhibited unexpected toxic side-effects, typified by pyrexia and hypertension $[16,17]$. Systemic application of TNF- $\alpha$ was, therefore, abandoned despite intratumoral administration of TNF$\alpha$ showing significant antitumor effects in phase I studies[5]. Similar in vivo drawbacks are also found in the clinical applications of other bioactive proteins[6]. Keeping these problems in mind, we have devised ways to improve the polymer-conjugation system using the TNF- $\alpha$ as a model protein[18-23]. In PEGylation of TNF- $\alpha$, the specific activity of PEGylated TNF- $\alpha$ decreased with the degree of PEG-modification (i.e., PEG modification rate). Additionally, when the PEG modification rates are same, the bioactivity of PEGylated TNF- $\alpha$ decreased with an increase in the molecular size of the attached PEG. Thus, when bioactive proteins, such as TNF- $\alpha$, are able to stably express their activities after binding to polymeric macromolecules, proper attention should be paid to minimize the loss in activity arising from the inhibition of their binding to the receptor molecules due to the steric hindrance posed by the polymeric modifier. By assessing the relationship between the molecular weight of PEG attached to TNF$\alpha$, degree of modification of PEG-modified TNF- $\alpha$, and their in vivo antitumor potency, we succeeded in markedly and selectively enhancing the antitumor potency of PEGylated-TNF- $\alpha$ over that of the native TNF- $\alpha$. Our results suggest that comprehensive analysis of the relationship between the degree of modification by polymer, molecular size, and specific activity could lead to the development of bioconjugated proteins with increased therapeutic value and decreased side effects.

These results suggest that PEGylation is a pragmatic approach for developing successful therapies with drugs, 
such as enzymes and antitumor agents. We believe that the technique of bioconjugating drugs to a polymeric carrier will indeed be an important technique for expanding the clinical applications of therapeutic proteins. In this context it is noteworthy that in recent years several research laboratories around the world have created similar polymer-conjugated bioactive proteins. As a result, PEGylated granulocyte-colony stimulating factor (PEG-G-CSF; PEG filgrastim), PEGylated interferon-alpha (PEG-IFN; PEGASYS, PEG-Intron), PEGylated asparaginase (PEG-Asp; ONCASPER), PEGylated adenosine deaminase (PEG-ADA; ADAGEN) and polystyrene-co-maleic acid-conjugated neocarzinostatin (SMANCS) have been developed, all of which showed marked improvement in therapeutic efficacy in comparison to their corresponding native forms[24-28].

\section{A new method for site-specific PEGylation}

As described above, PEGylation is limited by the frequent substantial loss of protein specific activity associated with polymer-conjugation. In addition, such bioconjugated proteins could also consist of positional isomers of polymeric modifiers at various sites, each one of which may have distinct activity and other characteristics. Random modification could produce a heterogeneous mixture of conjugated proteins, consisting of different number of modifier molecules bound to various sites of the protein. As a result, functional properties of the bioconjugated proteins (such as activation, in vivo behavior, stability) are compromised, and thus, they could exhibit inconsistent therapeutic effects. Therefore, to improve the polymer-conjugation technique in the post-genome era, the pharmaceutical technology would require a method that maintains the present efficiency of polymer modification while simultaneously making the site-specific modification possible. For example, mutant proteins with free cysteine residues were created for modification. However, introduction of cysteine residues led to protein misfolding and aggregation, resulting in unexpected loss of activity. Furthermore, even if it were possible to produce a cysteine mutant that retained activity, the poor efficiency of polymer conjugation to the thiol group made it impossible to obtain the desired product.

To overcome this problem of PEGylation, we have developed a strategy for site-specific PEGylation of TNF- $\alpha$ (Figure 2). Because a deletion mutant of TNF- $\alpha$ lacking eight residues at the $\mathrm{N}$ terminus retains full bioactivity, we surmised that the $\mathrm{N}$ terminus of TNF- $\alpha$ is not important for function and might therefore be a good target for PEGylation[29]. However, TNF- $\alpha$ contains six internal lysine residues and the amino groups of all six of them are also targets for PEGylation. Sitedirected mutagenesis analysis have shown that Lys11

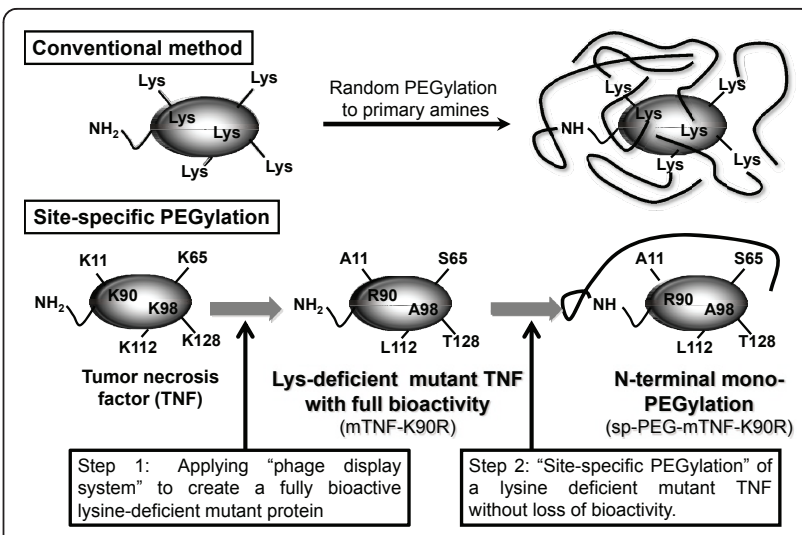

Figure 2 Site-specific PEGylation.

and Lys90 are vital for the bioactivity of TNF- $\alpha[30,31]$. Nevertheless, if one could construct fully bioactive TNF$\alpha$ in which all the lysine residues were replaced with other amino acids, site-specific PEGylation of the N-terminus could then be carried out. This PEGylated mutant TNF- $\alpha$ would be expected to have excellent molecular uniformity and retain high bioactivity.

To construct fully bioactive TNF- $\alpha$ in which all the lysine residues were replaced with other amino acids, we employed the "molecular evolution strategy" developed in our laboratory to artificially create functional muteins using our phage display system[32,33]. Phage libraries displaying polypeptides, such as naive antibodies or random peptides, have been extensively used for identifying specific molecules with high affinity for a target ligand [34-36]. The advantages of a phage display system are easy preparation of a library consisting of structural variants of a polypeptide as diverse as over one hundred million and isolation of several targeted ligand-binding molecules from this library in few weeks. There are, however, few studies in which the phage display technique has been used to create therapeutically useful structural variants of a bioactive protein, such as a mutein with stronger bioactivity and longer plasma half-life. To create a lysine-deficient mutant TNF- $\alpha$, a phage library displaying mutant TNF- $\alpha$ lacking any lysine residue was prepared, and it consisted of $<1 \times 10^{8}$ independent structural variants. After two rounds of biopanning against TNF-receptor, a lysine-deficient mutant TNF- $\alpha$, mTNF-K90R, with an in vitro bioactivity that was 6-fold stronger than that of the wild-type TNF- $\alpha$ (wTNF- $\alpha$ ) was obtained, despite reports that some of the lysine residues of TNF- $\alpha$ were essential for its bioactivity. This mTNF-K90R had $<10$-fold higher in vivo antitumor potency and 1.3-fold lower in vivo toxicity compared to those of wTNF- $\alpha$. Therefore, the therapeutic window of mTNF-K90R was extended by $<13$-fold more than that of the wTNF- $\alpha$. Whereas multiple PEG molecules 
attached randomly to various sites of wTNF- $\alpha$, only a single PEG molecule attached selectively to the $\mathrm{N}$-terminus of mTNF-K90R. The site-specific mono-PEGylated mTNF-K90R (sp-PEG-mTNF-K90R) exhibited 60\% specific activity of that of the mTNF-K90R, whereas the randomly mono-PEGylated wTNF- $\alpha$ (ran-PEG-wTNF$\alpha$ ) exhibited only $6 \%$ specific activity of that of the wTNF- $\alpha$ (Table. 1). Surprisingly, the sp-PEG-mTNFK90R showed higher in vitro bioactivity than the wTNF$\alpha$. Thus, these results suggest that by using this site-specific PEGylation method, we have been able to circumvent the problems associated with the random PEGylation method. The sp-PEG-mTNF-K90R exhibited an antitumor potency that was 3-fold, > 30-fold, and > 30 -fold higher than the antitumor potency exhibited by mTNF-K90R, wTNF- $\alpha$ and ran-PEG-wTNF- $\alpha$, respectively. In vivo toxicity of sp-PEG-mTNF-K90R was < 1.5-fold, 2.0-fold, and 0.6-fold lower than the in vivo toxicity exhibited by mTNF-K90R, wTNF- $\alpha$, and ranPEG-wTNF- $\alpha$, respectively. Thus, the therapeutic window of sp-PEG-mTNF-K90R expanded by > 5-fold, 60fold, and 18-fold than the therapeutic window of mTNF-K90R, wTNF- $\alpha$, and ran-PEG-wTNF- $\alpha$, respectively. These results clearly demonstrate the advantages of creating muteins and developing methods for sitespecific PEGylation to promote pharmacoproteomicbased protein-drug discovery and development.

Site-specific polymer-conjugation is possible even for a protein whose $\mathrm{N}$-terminal region plays an essential role in activation by making use of the differences between the reaction patterns of $\alpha$-Amino Groups and $\varepsilon$-Amino Groups, and this could be achieved by first creating a functional lysine-deficient mutant and then introducing a new lysine residue in an area that is not connected with activation.

\section{Functionalized polymer carriers as DDS}

In order to deliver a bioconjugated drug to the targeted tissue, the conjugate must demonstrate desirable pharmacokinetic characteristics, such as plasma clearance and tissue distribution. It is well known that the in vivo pharmacokinetics of polymer-conjugated drugs, such as bioactive proteins, could be markedly influenced by the

Table 1 In vitro bioactivity of mono-PEGylated forms of TNF- $\alpha$ s

\begin{tabular}{ll}
\hline & EC50 $(\mathbf{n g} / \mathbf{m l})(\%$ of remaining activity) \\
\hline WTNF & 0.17 \\
mTNF-K90R & 0.03 \\
ran-PEG-WTNF & $2.85(6.0 \%)$ \\
sp-PEG-mTNF-K90R & $0.05(60.0 \%)$ \\
\hline
\end{tabular}

The specific activity of the mono-PEGylated forms of TNF- $\alpha$ was measured by a cytotoxic assay using LM cells in the presence of actinomycin $D . E C_{50}$ is the concentration of various PEGylated TNF- $\alpha$ s capable of killing $50 \%$ of the cells. properties (such as electric charge and hydrophilic/ hydrophobic balance) of the polymeric carriers attached to the drug surface[37,38]. Any increase in the therapeutic effect of a drug bioconjugated with a polymeric modifier could be attributed to the pharmacokinetics of the bioconjugated drug. Therefore, prior to selecting a polymeric modifier for bioconjugation, it is very important to take into consideration the influence of physicochemical characteristics on the pharmacokinetics of the polymer. For example, we have demonstrated that for increased retention in blood, polyvinylpyrrolidone (PVP) is overwhelmingly superior to PEG as a polymer carrier [39].

These series of advances have also led us to successfully synthesize polyvinylpyrrolidone-co-dimethyl maleic anhydride (PVD), a renal-targeting polymer carrier with $\mathrm{pH}$-sensitive controlled release capability $[37,40]$. PVD attaches to the amino group of a protein at $\mathrm{pH} \geq 8$ and these PVD-protein conjugates gradually releases the proteins at $\mathrm{pH} \leq 7$ (Figure 3). Since the diseased tissues (including tissues with inflammation, cancer, etc.) generally have lower $\mathrm{pH}$ levels than the healthy ones, this means that PVD as a drug/protein carrier would usually respond to $\mathrm{pH}$ levels and release the bound drug/protein, but only in the diseased tissues. Amazingly, $80 \%$ of PVD administered intravenously to mice accumulated in the kidney in only a few hours and $40 \%$ of PVD remained in the kidney for 4-days (Figure 3). The PVD was selectively taken up by the epithelial cells of the renal tubule. It neither showed any sign of cytotoxicity nor did it cause any tissue damage in the kidney or elsewhere even when administered at a high dose. In addition, the PVD conjugate of anti-inflammatory protein superoxide dismutase (SOD) was found to be highly stable in vivo and it accumulated at high levels in the kidney following an

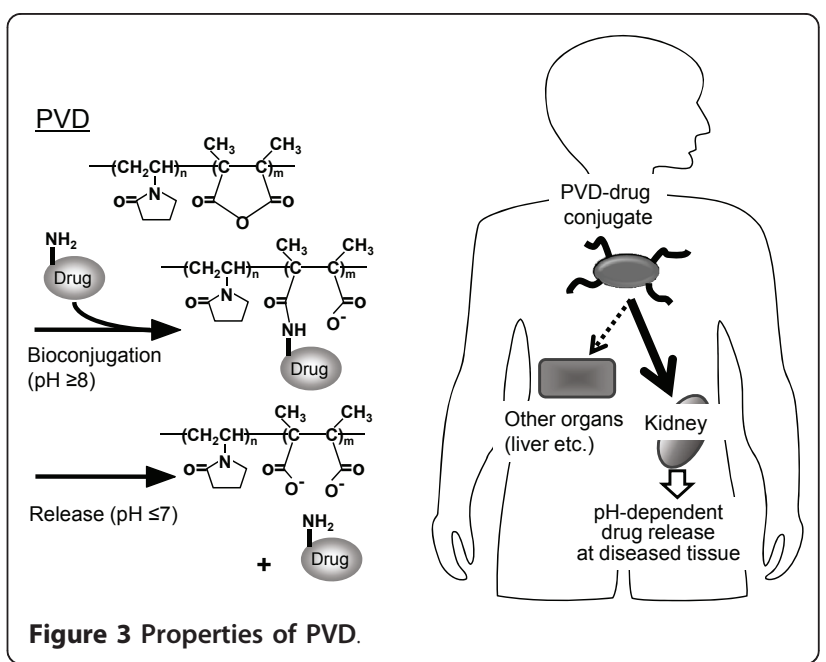


intravenous injection, bringing out the possibility that this conjugate may have significant therapeutic value in treating kidney inflammation.

There is no cure for renal disease, which is a serious problem on the rise world-wide, and very few preventive strategies are available against this disease. Bioactive proteins, such as SOD and IL-10, were thought to be able to prevent the progression of renal disease, but their therapeutic potencies were too low because they were poorly distributed in the kidney. Many researchers have made attempts to deliver drug to the kidney. However, so far, there is no available report on delivery of drugs specifically to the kidney. Development of a renal delivery system that selectively carries drugs to the kidney is a promising way of limiting the tissue distribution of drugs and controlling the drug-associated toxicity. Therefore, PVD may be a new and useful renal-targeting drug carrier, and PVD-SOD conjugate may find clinical applications in the effective treatment of various renal diseases.

\section{Conclusions}

In this review, we have demonstrated the usefulness of DDS-based technologies to selectively enhance the desirable therapeutic activities of bioactive proteins without increasing their side effects. Thus, we suggest that, to further promote the clinical applications of bioactive proteins, it is necessary for the protein-drug innovation system to combine 1) a method for creating mutant proteins (muteins) with desired properties and 2) a bioconjugation method that generates conjugated products with optimal properties, such as targeting capability. We believe that this protein-drug innovation system will be a valuable tool for the development of novel proteinbased therapies.

\section{Acknowledgements}

This study was supported in part by Grants-in-Aid for Scientific Research from the Ministry of Education, Culture, Sports, Science and Technology of Japan, and from the Japan Society for the Promotion of Science (JSPS). This study was also supported in part by Health Labour Sciences Research Grants from the Ministry of Health, Labor and Welfare of Japan; by Health Sciences Research Grants for Research on Publicly Essential Drugs and Medical Devices from the Japan Health Sciences Foundation; by a Global Environment Research Fund from Minister of the Environment; and by a the Knowledge Cluster Initiative; and by The Nagai Foundation Tokyo; and by The Takeda Science Foundation.

\footnotetext{
Author details

'Department of Toxicology and Safety Science, Graduate School of Pharmaceutical Sciences, Osaka University, 1-6 Yamadaoka, Suita, Osaka 5650871, Japan. ${ }^{2}$ The Center for Advanced Medical Engineering and Informatics, Osaka University, 1-6, Yamadaoka, Suita, Osaka 565-0871, Japan. ${ }^{3}$ Laboratory of Biopharmaceutical Research, National Institute of Biomedical Innovation, 7-6-8, Saito-Asagi, Ibaraki, Osaka 567-0085, Japan. ${ }^{4}$ Department of Biomedical Innovation, Graduate school of Pharmaceutical Sciences, Osaka University, 76-8 Saito-asagi, Ibaraki, Osaka 567-0085, Japan.
}

\section{Authors' contributions}

YY and ST wrote the manuscript. YT supervised the project. All authors discussed the results and commented on the manuscript.

\section{Competing interests}

The authors declare that they have no competing interests.

Received: 1 March 2011 Accepted: 12 May 2011 Published: 12 May 2011

\section{References}

1. Nishimoto N, Kishimoto T: Interleukin 6: from bench to bedside. Nat Clin Pract Rheumatol 2006, 2:619-626.

2. Pastan I, Hassan R, FitzGerald DJ, Kreitman RJ: Immunotoxin treatment of cancer. Annu Rev Med 2007, 58:221-237.

3. Szekanecz Z, Kerekes G, Soltesz P: Vascular effects of biologic agents in RA and spondyloarthropathies. Nat Rev Rheumatol 2009, 5:677-684.

4. Aghemo A, Rumi MG, Colombo M: Pegylated interferons alpha2a and alpha2b in the treatment of chronic hepatitis C. Nat Rev Gastroenterol Hepatol 2010, 7:485-494.

5. Kimura K, Taguchi T, Urushizaki I, Ohno R, Abe O, Furue H, Hattori T, Ichihashi $\mathrm{H}$, Inoguchi $\mathrm{K}$, Majima $\mathrm{H}$, et al: Phase I study of recombinant human tumor necrosis factor. Cancer Chemother Pharmacol 1987, 20:223-229.

6. Rosenberg SA, Lotze MT, Muul LM, Chang AE, Avis FP, Leitman S, Linehan WM, Robertson CN, Lee RE, Rubin JT, et al: A progress report on the treatment of 157 patients with advanced cancer using lymphokineactivated killer cells and interleukin-2 or high-dose interleukin-2 alone. N Engl J Med 1987, 316:889-897.

7. Yoshioka Y, Tsutsumi Y, Nakagawa S, Mayumi T: Recent progress on tumor missile therapy and tumor vascular targeting therapy as a new approach. Curr Vasc Pharmacol 2004, 2:259-270.

8. Pasut G, Veronese FM: PEG conjugates in clinical development or use as anticancer agents: an overview. Adv Drug Deliv Rev 2009, 61:1177-1188.

9. Maeda H: Tumor-selective delivery of macromolecular drugs via the EPR effect: background and future prospects. Bioconjug Chem 2010, 21:797-802.

10. Monkarsh SP, Ma Y, Aglione A, Bailon P, Ciolek D, DeBarbieri B, Graves MC, Hollfelder K, Michel H, Palleroni A, Porter JE, Russoman E, Roy S, Pan YC: Positional isomers of monopegylated interferon alpha-2a: isolation, characterization, and biological activity. Anal Biochem 1997, 247:434-440.

11. Bailon P, Palleroni A, Schaffer CA, Spence CL, Fung WJ, Porter JE, Ehrlich GK, Pan W, Xu ZX, Modi MW, Farid A, Berthold W, Graves M: Rational design of a potent, long-lasting form of interferon: a $40 \mathrm{kDa}$ branched polyethylene glycol-conjugated interferon alpha-2a for the treatment of hepatitis C. Bioconjug Chem 2001, 12:195-202.

12. Carswell EA, Old LJ, Kassel RL, Green S, Fiore N, Williamson B: An endotoxin-induced serum factor that causes necrosis of tumors. Proc Natl Acad Sci USA 1975, 72:3666-3670.

13. Blick M, Sherwin SA, Rosenblum M, Gutterman J: Phase I study of recombinant tumor necrosis factor in cancer patients. Cancer Res 1987, 47:2986-2989.

14. Abbruzzese JL, Levin B, Ajani JA, Faintuch JS, Saks S, Patt YZ, Edwards C, Ende K, Gutterman JU: Phase I trial of recombinant human gammainterferon and recombinant human tumor necrosis factor in patients with advanced gastrointestinal cancer. Cancer Res 1989, 49:4057-4061.

15. Creaven PJ, Plager JE, Dupere S, Huben RP, Takita H, Mittelman A, Proefrock A: Phase I clinical trial of recombinant human tumor necrosis factor. Cancer Chemother Pharmacol 1987, 20:137-144.

16. Chapman PB, Lester TJ, Casper ES, Gabrilove JL, Wong GY, Kempin SJ, Gold PJ, Welt S, Warren RS, Starnes HF, et al: Clinical pharmacology of recombinant human tumor necrosis factor in patients with advanced cancer. J Clin Oncol 1987, 5:1942-1951.

17. Sherman ML, Spriggs DR, Arthur KA, Imamura K, Frei E, Kufe DW: Recombinant human tumor necrosis factor administered as a five-day continuous infusion in cancer patients: phase I toxicity and effects on lipid metabolism. J Clin Oncol 1988, 6:344-350.

18. Tsutsumi Y, Kihira T, Yamamoto S, Kubo K, Nakagawa S, Miyake M, Horisawa Y, Kanamori T, Ikegami H, Mayumi T: Chemical modification of natural human tumor necrosis factor-alpha with polyethylene glycol increases its anti-tumor potency. Jpn J Cancer Res 1994, 85:9-12. 
19. Tsutsumi Y, Kihira T, Tsunoda S, Kubo K, Miyake M, Kanamori T, Nakagawa S, Mayumi T: Intravenous administration of polyethylene glycol-modified tumor necrosis factor-alpha completely regressed solid tumor in Meth-A murine sarcoma model. Jpn J Cancer Res 1994, 85:1185-1188.

20. Tsutsumi Y, Kihira T, Tsunoda S, Kanamori T, Nakagawa S, Mayumi T: Molecular design of hybrid tumour necrosis factor alpha with polyethylene glycol increases its anti-tumour potency. Br J Cancer 1995, 71:963-968.

21. Tsutsumi $Y$, Kihira T, Tsunoda S, Kamada H, Nakagawa S, Kaneda $Y$, Kanamori T, Mayumi T: Molecular design of hybrid tumor necrosis factoralpha III: polyethylene glycol-modified tumor necrosis factor-alpha has markedly enhanced antitumor potency due to longer plasma half-life and higher tumor accumulation. J Pharmacol Exp Ther 1996, 278:1006-1011.

22. Tsutsumi Y, Tsunoda S, Kamada H, Kihira T, Nakagawa S, Kaneda Y, Kanamori T, Mayumi T: Molecular design of hybrid tumour necrosis factor-alpha. II: The molecular size of polyethylene glycol-modified tumour necrosis factor-alpha affects its anti-tumour potency. $\mathrm{Br} J$ Cancer 1996, 74:1090-1095.

23. Tsutsumi $Y$, Tsunoda S, Kaneda $Y$, Kamada H, Kihira T, Nakagawa S, Yamamoto $Y$, Horisawa $Y$, Mayumi T: In vivo anti-tumor efficacy of polyethylene glycol-modified tumor necrosis factor-alpha against tumor necrosis factor-resistant tumors. Jpn J Cancer Res 1996, 87:1078-1085.

24. Hershfield MS: PEG-ADA replacement therapy for adenosine deaminase deficiency: an update after 8.5 years. Clin Immunol Immunopathol 1995, 76:S228-232

25. Chapes SK, Simske SJ, Sonnenfeld G, Miller ES, Zimmerman RJ: Effects of spaceflight and PEG-IL-2 on rat physiological and immunological responses. J Appl Physiol 1999, 86:2065-2076.

26. Maeda H: SMANCS and polymer-conjugated macromolecular drugs: advantages in cancer chemotherapy. Adv Drug Deliv Rev 2001, 46:169-185.

27. Talpaz M, O'Brien S, Rose E, Gupta S, Shan J, Cortes J, Giles FJ, Faderl S, Kantarjian HM: Phase 1 study of polyethylene glycol formulation of interferon alpha-2B (Schering 54031) in Philadelphia chromosomepositive chronic myelogenous leukemia. Blood 2001, 98:1708-1713.

28. Isidori A, Tani M, Bonifazi F, Zinzani P, Curti A, Motta MR, Rizzi S, Giudice V, Farese O, Rovito M, Alinari L, Conte R, Baccarani M, Lemoli RM: Phase II study of a single pegfilgrastim injection as an adjunct to chemotherapy to mobilize stem cells into the peripheral blood of pretreated lymphoma patients. Haematologica 2005, 90:225-231.

29. Jones EY, Stuart DI, Walker NP: Structure of tumour necrosis factor. Nature 1989, 338:225-228

30. Yamagishi J, Kawashima H, Matsuo N, Ohue M, Yamayoshi M, Fukui T, Kotani H, Furuta R, Nakano K, Yamada M: Mutational analysis of structureactivity relationships in human tumor necrosis factor-alpha. Protein Eng 1990, 3:713-719.

31. Van Ostade $X$, Tavernier J, Prange $T$, Fiers W: Localization of the active site of human tumour necrosis factor (hTNF) by mutational analysis. EMBO J 1991, 10:827-836.

32. Yamamoto $Y$, Tsutsumi Y, Yoshioka Y, Nishibata T, Kobayashi K, Okamoto T, Mukai Y, Shimizu T, Nakagawa S, Nagata S, Mayumi T: Site-specific PEGylation of a lysine-deficient TNF-alpha with full bioactivity. Nat Biotechnol 2003, 21:546-552.

33. Shibata H, Yoshioka Y, Ikemizu S, Kobayashi K, Yamamoto Y, Mukai Y, Okamoto T, Taniai M, Kawamura M, Abe Y, Nakagawa S, Hayakawa T, Nagata S, Yamagata Y, Mayumi T, Kamada H, Tsutsumi Y: Functionalization of tumor necrosis factor-alpha using phage display technique and PEGylation improves its antitumor therapeutic window. Clin Cancer Res 2004, 10:8293-8300.

34. Nielsen UB, Marks JD: Internalizing antibodies and targeted cancer therapy: direct selection from phage display libraries. Pharm Sci Technolo Today 2000, 3:282-291.

35. Ruoslahti E: Targeting tumor vasculature with homing peptides from phage display. Semin Cancer Biol 2000, 10:435-442.

36. Mukai Y, Yoshioka Y, Tsutsumi Y: Phage display and PEGylation of therapeutic proteins. Comb Chem High Throughput Screen 2005, 8:145-152.

37. Kaneda Y, Tsutsumi Y, Yoshioka Y, Kamada H, Yamamoto Y, Kodaira H, Tsunoda S, Okamoto T, Mukai Y, Shibata H, Nakagawa S, Mayumi T: The use of PVP as a polymeric carrier to improve the plasma half-life of drugs. Biomaterials 2004, 25:3259-3266.
38. Yoshioka $Y$, Tsutsumi $Y$, Ikemizu S, Yamamoto $Y$, Shibata H, Nishibata T, Mukai Y, Okamoto T, Taniai M, Kawamura M, Abe Y, Nakagawa S, Nagata S, Yamagata Y, Mayumi T: Optimal site-specific PEGylation of mutant TNFalpha improves its antitumor potency. Biochem Biophys Res Commun 2004, 315:808-814

39. Kamada H, Tsutsumi $Y$, Yamamoto $Y$, Kihira T, Kaneda $Y$, Mu Y, Kodaira H, Tsunoda Sl, Nakagawa S, Mayumi T: Antitumor activity of tumor necrosis factor-alpha conjugated with polyvinylpyrrolidone on solid tumors in mice. Cancer Res 2000, 60:6416-6420.

40. Kamada H, Tsutsumi Y, Sato-Kamada K, Yamamoto Y, Yoshioka Y, Okamoto T, Nakagawa S, Nagata S, Mayumi T: Synthesis of a poly (vinylpyrrolidone-co-dimethyl maleic anhydride) co-polymer and its application for renal drug targeting. Nat Biotechnol 2003, 21:399-404

doi:10.1186/1752-153X-5-25

Cite this article as: Yoshioka et al:: Development of a novel DDS for sitespecific PEGylated proteins. Chemistry Central Journal 2011 5:25.

Publish with ChemistryCentral and every
scientist can read your work free of charge
"Open access provides opportunities to our
colleagues in other parts of the globe, by allowing
anyone to view the content free of charge."
W. Jeffery Hurst, The Hershey Company.
- available free of charge to the entire scientific community
- cited in PubMed and archived on PubMed Central
- yours - you keep the copyright
Submit your manuscript here:
http://www.chemistrycentral.com/manuscript/

